\title{
Novel Resonant Structure to Compact Partial H-Plane Band-Pass Waveguide Filter
}

\author{
Elahe Mohammadi, Habib Ghorbaninejad
}

Department of Electrical Engineering, University of Guilan, Iran

\begin{tabular}{l}
\hline \hline Article Info \\
\hline Article history: \\
Received Sep 26, 2016 \\
Revised Nov 20, 2016 \\
Accepted Dec 11, 2016 \\
\hline
\end{tabular}

\section{Keyword:}

Band-pass filter

Partial H-plane waveguide

Partial H-plane waveguide filter

Resonant structure

\begin{abstract}
In this paper partial H-plane band-pass waveguide filter, utilizing a novel resonant structure comprising a metal window along with metal posts has been proposed to compactthe filter size. The metal windows and postshave been implemented transversely in a partial $\mathrm{H}$-plane waveguides, which have one-quarter cross section size compared to the conventional waveguides in the same frequency range. Partial H-plane band-pass waveguide filter with novel proposed resonant structures has considerably shorter longitudinal length compared to the conventional partial H-plane filters, so that they reduce both cross section size and the total length of the filter compared to conventional H-plane filters, in the same frequency range. In the presented design procedure, the size and shape of each metal window and metal posts has been determined by fitting the transfer function of the proposed resonant structure to that of a desiredone, which is obtained from a suitable equivalent circuit model. The design process is based on optimization using electromagnetic simulator software, HFSS. A proposed partial H-plane bandpass filter has been designed and simulated to verify usefulness and performanceof the design method.
\end{abstract}

Copyright $\odot 2017$ Institute of Advanced Engineering and Science. All rights reserved.

\section{Corresponding Author:}

Habib Ghorbaninejad,

Department of Electrical Engineering,

University of Guilan,

Rasht, Guilan Province, Iran.

Email: ghorbaninejad@guilan.ac.ir

\section{INTRODUCTION}

Microwave filters are of the most important components in RF/Microwave systems that can be used in many devices such as satellite communication equipment. Fast development of RF technology requires compact, low-cost and mass-producible properties for its components. Inductive elements such as irises, rods, diaphragms and postshave been used in conventional filters in which hollow or filled transmission line act as resonators [1-6]. The dielectric resonator (DR) is a type of resonator, whichhas been introduced to compact overall size of band-pass filters [7-10], but they lead to dielectric loss. E-plane filters are low loss, easy to fabricate, low-cost and mass-producible, but despite their desired characteristics, they suffer disadvantage of large volumes, especially in low frequencies [11]. In addition, H-plane filters [12] are widely used in the design of microwave filters but they have large sizes that are a deficiency for such as filters. The design method of partial H-plane band-pass filter with evanescent and transmission sections of partial H-plane waveguide is extensively explained in which is based on optimization using an electromagnetic simulation software [12].

The dispersion characteristic of both H-plane waveguide and conventional waveguide are the same for first and second mode, while the cross section of a partially H-plane waveguide is one quarter of the cross section of a conventional waveguide [13]. So, using partially H-plane waveguide instead of conventional one reduce the cross section size of the filter to one quarter. Since in both filters the resonators are realized by 
half-wavelength waveguide sections, there is not any reduction in the total longitudinal length. To further reduction of the longitudinally size of the filter, a type of partial H-plane filter using quarter wavelength resonators is designed by which the length of filter is reduced to that of conventional E-plane filter about $29.2 \%$ [13].

In this paper a novel partial H-plane band-pass waveguide filter using a novel resonant structure are designed so that its longitudinally length is considerably shorter than that of half wavelength or quarter wavelength waveguide sections which is used in the conventional partial H-plane filters. The novel resonant structure is composed of a transverse metal window along with metal posts to considerably reduce the longitudinal size of the filter. If half wavelength or quarter wavelength waveguide sections replace by the proposed novel resonator structure, the longitudinally size of the filter will be reduced while its cross section is one quarter ofconventional waveguide filters. Configuration of the proposed resonator structures is determined by fitting the transfer function of a desired oneto that calculated from an equivalent circuit model.Here, compact resonators are designed in partial H-plane filters whose lengths are considerably shorter. Since this type of resonators is transversely placed in the partial H-plane waveguide leads to further size reduction in thefilter structure.

\section{PARTIAL H-PLANE WAVEGUIDE}

Figure 1 shows the configuration of a typical partial H-plane waveguide and its cross section (front) view. Partial H-plane waveguide is the same conventional rectangular waveguide in which a H-plane metal vane is partially placed. In the same frequency range, the cross section of partially H-plane waveguide is one quarter of the conventional one. To obtain a partially H-plane waveguide, one should transversely fold a rectangular waveguide. It has been shown that the dispersion characteristics of partial H-plane waveguide of size $\mathrm{a}=23.8 \mathrm{~mm}, \mathrm{~b}=12 \mathrm{~mm}, \mathrm{~d}=19.7 \mathrm{~mm}$ and conventional rectangular waveguide of size $\mathrm{a}=47.55 \mathrm{~mm}$, $b=22.15 \mathrm{~mm}$ in the frequency range of $2-8 \mathrm{GHz}$, for dominant and second mode are the same. The dimensions show that the cross section of partial H-plane waveguide is one quarter of that of the conventional one.
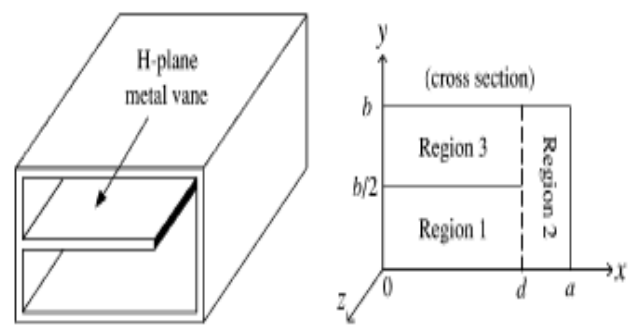

Figure 1. Partial H-plane waveguide and its cross section [13]

\section{THE PROPOSED RESONANT STRUCTURE CONFIGURATION}

Figure 2(a) shows the configuration of the proposed resonant structure which is placed in a partial H-plane waveguide. Resonant structure is composed of a metal window along with metal posts. Figure 2(b), (c) depict front view and top view of the proposed resonant structure, respectively.

\section{PARTIAL H-PLANE WAVEGUIDE BAND-PASS FILTER USING THE PROPOSED RESONANT STRUCTURE}

Figure 3 shows the three dimensional view of a partial H-plane waveguide band pass filter using the proposed resonant structures. The presented configuration is composed of three proposed resonant structure, which is placed in the partial H-plane waveguide so that the length of interval between them is one-quarter wavelength $\left(\lambda_{\mathrm{g}} / 4\right)$. One-quarter wavelength sections act as inverters. Based on symmetry condition for Chebyshev band pass filter, two resonant structures with equal distance from the symmetry plane are the same. In the introduced filter, partial H-plane waveguide sections with one-quarter length act as impedance inverters. In the proposed designed method the length (thickness) of each resonator is negligible; therefore it is longitudinally shorter than any resonant structure such as half wavelength waveguide section or even quarter wave length waveguide section, resulting in longitudinally reduced size filters. 


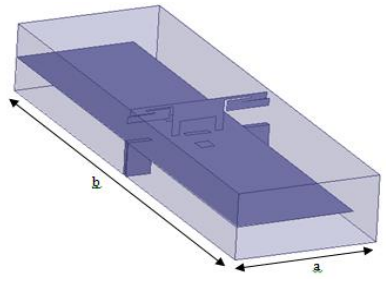

(a)

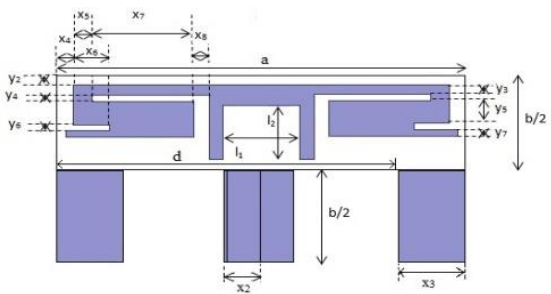

(b)

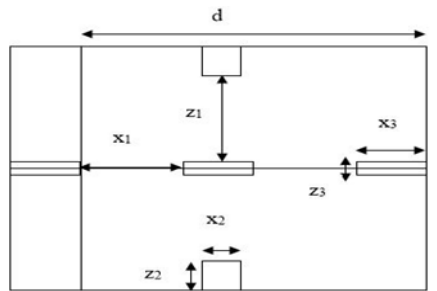

(c)

Figure 2. The proposed resonant structure, (a) 3D view, (b) front view, and (c) top view

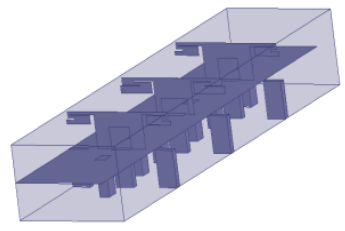

Figure 3. Partial H-plane band-pass waveguide filter with designed proposed resonator structures which are placed in interval s of the length $\ell=19.4 \mathrm{~mm}$

\section{FILTER DESIGN METHOD}

To design a typical waveguide band-pass filter, at the first step, an LC equivalent circuit model should be provided. Figure 4 depicts a typical band pass filter consisting of $\mathrm{N}$ parallel LC resonator and $\mathrm{N}+1$ admittance inverters. At the second step, the parameters of equivalent circuit model, including the values of admittance invertors, $\mathrm{L}$ and $\mathrm{C}$ values of parallel resonators can be calculated according to the desired (given) filter characteristics, such as type of the filter, operating center frequency and the filter bandwidth. In this work, the equivalent circuit model has been realized using $\mathrm{N}$ shunt resonators and $\mathrm{N}+1$ quarter wavelength transmission line sections as admittance inverters. At the final step at which, all the parameters of equivalent circuit model are obtained, it is aimed to realize each of LC resonators by a suitable configuration of the proposed resonant structure. For this purpose, and in order to obtain suitable configuration, the various parameters of the resonant structure, are optimized using an electromagnetic simulator (HFSS) so that the transfer function of the resonant structure can be fitted to that of LC equivalent circuit model ones. In the optimization process the defined parameters of each proposed resonant structure are obtained so that the scattering parameters of electromagnetic simulator fit to that of LC circuit model, at an acceptable number of frequency samples. After obtaining the configuration of all resonators, they will be located in the partial $\mathrm{H}-$ plane waveguide in a way that the length between interval resonators is equal to one quarter of wavelength. So, the basic step at the design process is obtaining the configuration of each resonator, the next section will be dedicated to design details of the proposed resonant structure. The later section dedicated to the design method of compact resonators using high frequency simulation software and circuit theory model.

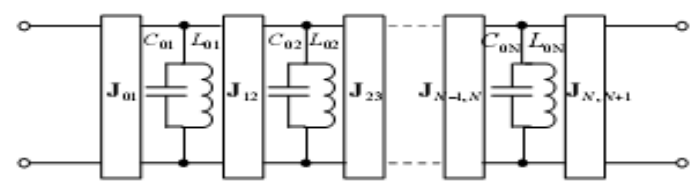

Figure 4. Typical band pass filter using parallel LC resonator and admittance inverters

\section{THE PROPOSED RESONANT STRUCTURE DESIGN}

The design method of the proposed resonant structure is based on optimization process that can be done using a high frequency structure simulator, here, HFSS. For a given specifications of desired filter, the values of $\mathrm{L}$ and $\mathrm{C}$ of each circuit model resonator can be easily determined and in turn transmission coefficient of resonators will be determined in the frequency range. HFSS software optimizes the geometrical parameters of the proposed resonant structure so that its transmission coefficient fit to that of circuit model 
one. To design a 3-order equal ripple Chebyshev filter with center frequency $\mathrm{f}_{0}=5 \mathrm{GHz}$, the relative bandwidth $16 \%$ and equal ripples $0.8 \mathrm{~dB}$, the value of circuit model resonators are, $\mathrm{L}_{01}=\mathrm{L}_{03}=1.25 \mathrm{nH}$, $\mathrm{C}_{01}=\mathrm{C}_{03}=0.805 \mathrm{pF}, \mathrm{L}_{02}=107.7 \mathrm{nH}, \mathrm{C}_{02}=0.0093 \mathrm{pF}$. Due to symmetry conditions, resonators 1 and 3 are the same.

In this work for each optimization process, frequency step is assumed to be $10 \mathrm{MHz}$, so 201 frequency samples in the range of frequency domain, $4-6 \mathrm{GHz}$ is considered. The optimized values are given in table 1 for the resonators. Partial H-plane waveguide which is used in this design, utilizes a rectangular waveguide of dimension $a=23.8 \mathrm{~mm}, \mathrm{~b}=12 \mathrm{~mm}$ and a partial $\mathrm{H}$-plane metal vane of width, $\mathrm{d}=19.7 \mathrm{~mm}$ Thickness of themetal vane has been considered to be $1 \mathrm{~mm}$. Figure 5 shows transmission coefficient of LC circuit model and that of the proposed resonant structures. There is a relatively good agreement between the frequency characteristics of desired LC resonators and that of the proposed one over the frequency range. Table 1 shows geometrical parameters of the optimized proposed resonant structures (in $\mathrm{mm}$ ).

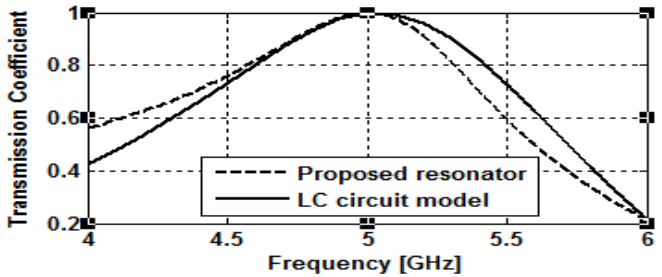

(a)

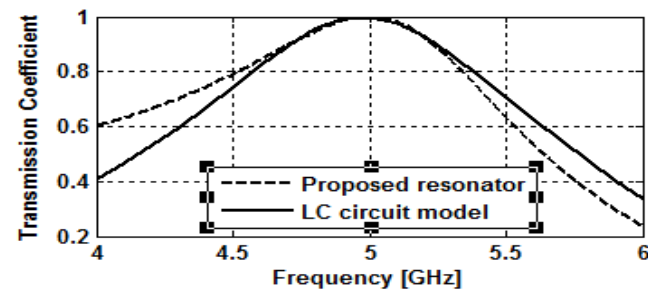

(b)

Figure 5. Transmission coefficient of LC circuit model and that of the proposed resonant structures, (a) resonators $1 \& 3$, (b) resonator (2)

Table 1. Geometrical Parameters of the Optimized Proposed Resonant Structures (in mm)

\begin{tabular}{|c|c|c|c|c|c|c|c|c|c|c|c|c|c|}
\hline Global variables & $\begin{array}{c}\mathrm{a} \\
23.8 \\
\end{array}$ & $\begin{array}{c}\mathrm{b} \\
12\end{array}$ & $\begin{array}{c}d \\
19.7\end{array}$ & $\begin{array}{c}\mathrm{X}_{1} \\
5.9 \\
\end{array}$ & $\begin{array}{c}\mathrm{X}_{2} \\
2.21 \\
\end{array}$ & $\begin{array}{c}\mathrm{X}_{3} \\
4 \\
\end{array}$ & $\begin{array}{c}\mathrm{y}_{1} \\
5.999\end{array}$ & $\begin{array}{c}\mathrm{Z}_{1} \\
6.5 \\
\end{array}$ & $\begin{array}{c}Z_{2} \\
2.21 \\
\end{array}$ & $\begin{array}{c}\mathrm{Z}_{3} \\
1 \\
\end{array}$ & & & \\
\hline Resonator no. & $\mathrm{X}_{4}$ & $\mathrm{X}_{5}$ & $\mathrm{X}_{6}$ & $\mathrm{x}_{7}$ & $\mathrm{X}_{8}$ & $\mathrm{y}_{2}$ & $\mathrm{y}_{3}$ & $\mathrm{y}_{4}$ & $\mathrm{y}_{5}$ & $\mathrm{y}_{6}$ & $\mathrm{y}_{7}$ & $\mathrm{l}_{1}$ & $l_{2}$ \\
\hline $1 \& 3$ & 0.45 & 0.49 & 1.4 & 4.5 & 0.98 & 0.13 & 0.45 & 0.1 & 0.4 & 0.38 & 0.66 & 4.5 & 7 \\
\hline 2 & 0.48 & 2.61 & 4 & 4 & 0.53 & 0.2 & 0.48 & 0.23 & 0.5 & 0.07 & 1.2 & 5.93 & 5.08 \\
\hline
\end{tabular}

\section{EXAMPLE AND RESULTS}

To verify the design process, in this section the proposed resonant structures which have been optimized in former section, is placed in the partial $\mathrm{H}$-plane waveguide to achieve band pass waveguide filter with assumed specifications. The length interval between resonators is equal to $\lambda_{\mathrm{g}} / 4=19.4 \mathrm{~mm}$. Figure 6 shows transmission coefficient of the final Partial H-plane waveguide band pass filter, using the obtained resonant structures. Also the transfer coefficient of the desired filter has been shown in the figure. It can be deduced that there is a relatively good agreement between the transmission coefficients of the proposed filter compared to that of desired one. A slight frequency shift to higher frequencies can be observed that may be due to detuning of each resonator at the center frequency. Here, the coupling effect between resonators is weak, so it has not been considered in the design process, but in the case in which the coupling effect is dominant, the optimization process should be performed again for the whole structure in the frequency range to take in account the strong coupling effect.

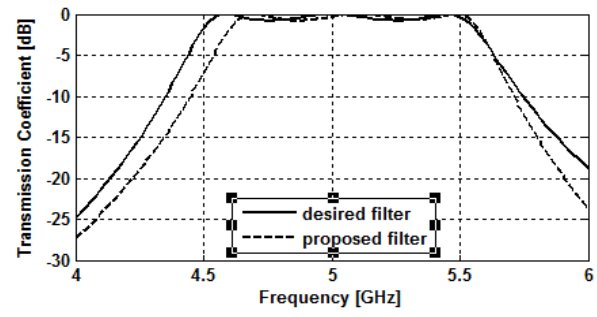

Figure 6. Transmission coefficient of thepartial H-plane waveguide band pass filter using the proposed resonators and that of the desired one 


\section{CONCLUSION}

In this paper a novel resonant structure has been proposed. The obtained resonators have been located in the partial H-plane waveguide to introduce partial H-plane band pass waveguide filter. Geometrical parameters of the proposed resonators have been obtained using optimization by HFSS software. Total length of the designed filter is $38.8 \mathrm{~mm}(2 \times 19.4 \mathrm{~mm})$, whilethe length of partial H-plane filters with half wavelength or quarter wavelength resonators is $144.11 \mathrm{~mm}$ in the bestcase [13]. The proposed resonator structure, has considerably reduced overalllongitudinally length of the filter, while its cross section is the same as conventional partial H-plane waveguide filter which is one quarter of the cross section of the conventional waveguide filter. The simulated has been performed to verify the design process.

\section{REFERENCES}

[1] Rahimian, Ardavan. "Modeling and Performance of Microwave and Millimeter-Wave Layered Waveguide Filters". Indonesian Journal of Electrical Engineering and Computer Science. 11.7 (2013): 3523-3533.

[2] Zhang, Pingjuan, and Minquan Li. "A Novel Compact Microstrip Lowpass Filter with Sharp Transition and Improved Stopband". Indonesian Journal of Electrical Engineering and Computer Science. 13.1 (2015): 85-90.

[3] Matthaei GL, Young L, Jones EM. Microwave filters, impedance-matching networks, and coupling structures. Artech house; 1980.

[4] Pozar DM. Microwave engineering. John Wiley \& Sons; 2009 Sep 1.

[5] Colline RE. Foundation for Microwave Engineering. McGraw Hill, New York. 1992.

[6] Levy R, Snyder RV, Matthaei G. Design of microwave filters. IEEE Transactions on Microwave Theory and techniques. 2002 Mar; 50(3): 783-93.

[7] Khalaj-Amirhosseini M. Microwave filters using waveguides filled by multi-layer dielectric. Progress In Electromagnetics Research. 2006; 66: 105-10.

[8] Ghorbaninejad H, Khalaj-Amirhosseini M. Compact bandpass filters utilizing dielectric filled waveguides. Progress In Electromagnetics Research B. 2008; 7: 105-15.

[9] Piloni M, Ravenelli R, Guglielmi M. Resonant aperture filters in rectangular waveguide. In Microwave Symposium Digest, 1999 IEEE MTT-S International. 1999 Jun 13 (Vol. 3, pp. 911-914). IEEE.

[10] Capurso M, Piloni M, Guglielmi M. Resonant aperture filters: Improved out-of-band rejection and size reduction. In Microwave Conference, 2001. 31st European. 2001 Sep 24 (pp. 1-4). IEEE.

[11] Postoyalko V, Budimir DS. Design of waveguide E-plane filters with all-metal inserts by equal ripple optimization. IEEE transactions on microwave theory and techniques. $1994 \mathrm{Feb}$; 42(2): 217-22.

[12] Kozakowski P, Mrozowski M. Gradient-based optimization of filters using FDTD software. IEEE microwave and wireless components letters. 2002; 12(10): 389-91.

[13] Kim D.W., Kim D.J., Lee J.H. Compact Partial H-Plane Filters. IEEE Trans. On Microw. Theory Tech. 2006 Nov; 54(11): 3923-30.

\section{BIOGRAPHIES OF AUTHORS}

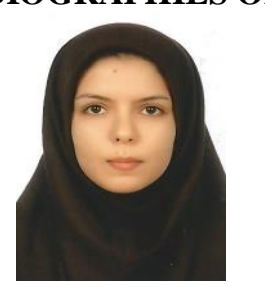

E. M. Variani received B.Sc. degree in Electrical Engineering from Isfahan University of Technology (IUT), Isfahan, Iran in 2012 and is currently working toward the M.S. degree in Fields and Wave Communication Engineering at University of Guilan. She has worked as a R\&D engineer in Mehad Sanat, Karaj, Iran since 2013.

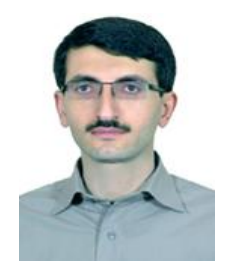

H. Ghorbaninejad received B. Sc. degree from University of Guilan in 2003 and M. Sc. and Ph. D. degrees from Iran University of Science and Technology (IUST) in 2005 and 2010 respectively, all in Fields and Wave Communication Engineering. He is currently assistant professor at Department of Electrical Engineering of University of Guilan. His scientific fields of interest are electromagnetic problems including microwave filter design, compact microwave devices and Green's function of microwave structures. 